\title{
DESIGN OF METAHEURISTIC OPTIMIZATION FOR CLUSTER BASED RELIABLE CRITICAL MESSAGE DISSEMINATION IN VEHICULAR ADHOC NETWORKS
}

\author{
S. Parameswari \\ Research Scholar, \\ Department of Computer Science and Engineering, Annamalai University, \\ Annamalai Nagar-608 002. \\ eswari105@gmail.com \\ Dr. K. Kavitha \\ Research Supervisor, Associate Professor \\ Department of Computer Science and Engineering, Annamalai University, \\ Annamalai Nagar-608 002. \\ kavithacseau@gmail.com \\ Dr. P. Elango \\ Dept. of Information Technology, \\ Perunthalivar Kamarajar Institute of Engineering and Technology (PKIET), \\ Karaikal-609603. \\ elanalin74@gmail.com
}

\begin{abstract}
Safety applications in Vehicular Adhoc Networks (VANET) are based on the dissemination of critical messages (CLMs) to numerous vehicles with minimal latency and packet loss. But the avoidance of broadcast storms and dealing with large scale dissemination of EMs in urban VANETs, predominantly at intersections, are challenging issues. These issues can be resolved by the clustering approach which can create a hierarchical structure by the organization of the nodes. In this view, this paper designs a metaheuristic optimization algorithm for cluster based reliable critical message dissemination (MOCRCMD) in VANET. The proposed MOC-RCMD technique aims to organize the clusters and select CHs proficiently. In addition, the MOC-RCMD technique encompasses a two-stage process namely Shark Optimization Algorithm based clustering (SOA-C) and Seagull Optimization based Routing for Data Dissemination (SGO-RDD). The SOA-C and SGO-RDD techniques derive a fitness function involving different input parameters related to VANET. The design of MOC-RCMD technique for cluster-based CM dissemination shows the novelty of the study. A wide range of simulations take place and the results demonstrated the betterment of the MOC-RCMD technique over the recent approaches.
\end{abstract}

Keywords: VANET, Metaheuristics, Clustering, Data dissemination, Critical Messages, Routing.

\section{Introduction}

Vehicular Adhoc Network (VANET) has significantly enhanced transportation system and helps in decreasing many redundant scenarios affecting irreversible mishaps on the road. VANET allows vehicles for communicating with each other either in parking areas/on roads. It enables vehicles to form a network of their own without centralized servers [1]. Each communication that takes place amongst vehicles is called vehicle to vehicle (V2V) transmission when the exchange of data among roadside units (RSUs) and vehicles are called vehicle to infrastructure (V2I) transmission. Practically, transmission frequency employed for vehicular information exchange in VANET is in the range of 5.85 and $5.925 \mathrm{GHz}$ [2]. Control channel (CC) and Service channel (SCH) are employed to transmit security messages based on crashes, accidents, and further hazardous activities, in addition, average data messages except for security messages. Although VANET is coupled with numerous problems data dissemination is a significant one. Generally, average messages aren't time crucial, unlike, emergency or safety messages are time crucial and require effective transmissions at the beginning [3]. There are distinct methods for dissemination of emergency messages (EM), but, most of them are developed for 


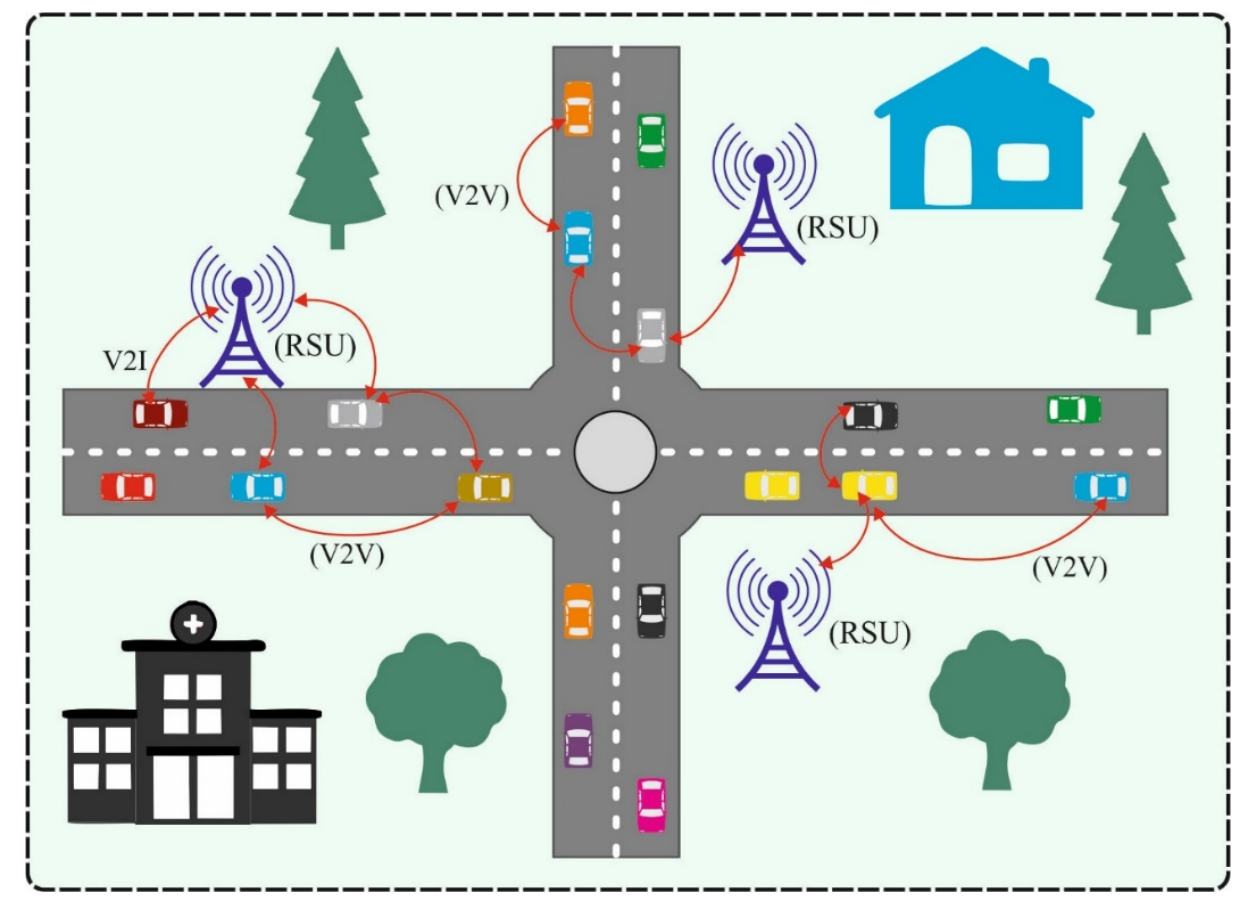

Fig. 1. VANET structure

working under certain scenarios. Still, there is room for considerable improvement since the technologies are yet developing and haven't reached their maturity. Safety application in VANET is based mainly on disseminating Emergency Messages (EM) [4]. Vehicles facing hazardous disseminate EM to another vehicle (hereafter nodes) within their transmission area. Fig. 1 illustrates the framework of VANET. It allows nodes to take necessary safety precautions, like travel delays, re-routing, traffic congestion, and avoid road accidents, [5]. In VANET, the more commonly used and effective means of disseminating EMs are flooding, where a source nodes broadcast EM to another node within its communication Range (R). Consecutively, the receiving node broadcasts EM in their R till the EM propagates over the entire network. But, owing to the dynamic nature of VANET, flooding cause broadcasts storms [6]. Consequently, the unnecessary communication of EM causes high delay, degrade the message reliability, and transmission congestion. For these reasons, several approaches like counter based and distance based disseminations, and Store-Carry-Forward (SCF) have been developed in the survey [7]. Nonetheless, SCF cause higher End-to-End (E2E) delays whereas counter based and distance based approaches are appropriate only for effective network. Furthermore, with no deployment of a centralized coordinator unit, the threats of redundant rebroadcasting can be increased. Subsequently, packet loss rate and E2E delay could also increase, particularly in higher density scenarios. This problem could be tackled by taking into account a cluster based method that could determine a network hierarchy by arranging nodes according to certain predetermined rules [8]. All the clusters have coordinator units, recalled a Cluster Head $(\mathrm{CH})$. Rather than, the re-transmitting nodes in a cluster deliver the information to its $\mathrm{CH}$ for additional dissemination. This approach could efficiently alleviate broadcast storms and communication congestion. But, node clustering in VANET has many open challenges like signal fades from adjacent nodes, mobility, non-uniform node distribution, and new challenges, along with overheads in cluster formation [9].

This paper designs a metaheuristic optimization algorithm for cluster based reliable critical message dissemination (MOC-RCMD) in VANET. The proposed MOC-RCMD technique aims to organize the clusters and select CHs proficiently. In addition, the MOC-RCMD technique encompasses a two-stage process namely Shark Optimization Algorithm based clustering (SOA-C) and Seagull Optimization based Routing for Data Dissemination (SGO-RDD). The SOA-C and SGO-RDD techniques derive a fitness function involving different input parameters related to VANET. The design of MOC-RCMD technique for cluster-based CM dissemination shows the novelty of the study. In order to showcase the better performance of the proposed model, a series of experimental analyses are carried out and the results are investigated interms of several measures.

\section{Literature Review}

Ullah et al. [10] introduced an EM dissemination system which depends on congestion avoidance scenarios in VANET and vehicular FoG computing. In the same way, FoG enabled VANET framework is examined which might effectively handle the message congestion scenario. Also, they present a taxonomy of schemes which tackle message congestion avoidances. Benkerdagh and Duvallet [11] presented a novel approach that includes data management beforehand dissemination processes as the initial phase of this system. In this stage, the original 
messages are enhanced for reducing the number of exchanged packets. Next, this proposition includes making stable and fast clusters for improving the messages transfer time also to obtain effective bandwidth consumptions. This method depends on a FF model that considered distinct parameters like link lifetime validity, relative velocity, connectivity degree, and transmission period.

Shah et al. [12] proposed data dissemination techniques with a time barrier method for reducing the overheads of the message which might clutter the network. The presented method depends on the concepts of a super-node to disseminate the message in timely manner. Furthermore, to evade redundant transmission that might affect the broadcast storms challenge, time barrier techniques are adopted for handling these problems. Therefore, only the distant vehicle rebroadcast the messages that might cover larger distance. Ali et al. [13] proposed a data dissemination system for this message in vehicular networks, according to the clustering and position based transmission technique. The vehicle is dynamically clustered for handling the transmission storm problems, and a position based method is presented for reducing the transmission delay, results in dissemination of Ems in timely manner.

Liu et al. [14] proposed a new TCEMD method that integrates the entity oriented trust value to data oriented trust evaluation in an effective way. In the presented method, while an emergency event (i.e., an obstacle in front of the road) happens, the Ems could be disseminated amongst the adjacent vehicles in a trust cascading method, in which the entity oriented trust value (that is updated and evaluated by leveraging the trust certificate and included in the message) is adapted as significant weight. Ullah et al. [15] proposed a clustering method for RBOEM method. The presented method depends on the mobility metrics to evade transmission overheads, retain the message reliability, and strengthen the cluster structure in higher mobility scenarios.

Ullah et al. [16] proposed an EEMDS method for urban VANET. The system depends on mobility metrics for avoiding transmission overheads and to retain a stable cluster formation. Each vehicle considered its path loss factor and direction angle for electing an appropriate CHs. Furthermore, they present evaluated connection stability to select appropriate relay vehicles which reduce the numbers of communication and rebroadcast congestion in the network. Arora [17] introduces a unique HPADD model by separating the road into a hybrid method. The election of relay vehicles is tackled for providing minimal end to end delays.

\section{The Proposed Model}

In this study, a new MOC-RCMD technique is derived to disseminate CLM in VANET. The proposed MOCRCMD technique involves a two-stage process namely SOA-C based cluster construction and SGO-RDD based Data Dissemination. The SOA-C and SGO-RDD techniques derive a fitness function involving different input parameters related to VANET. Fig. 2 demonstrates the overall workflow of MOC-RCMD model. The detailed processes are elaborated in the following sections.

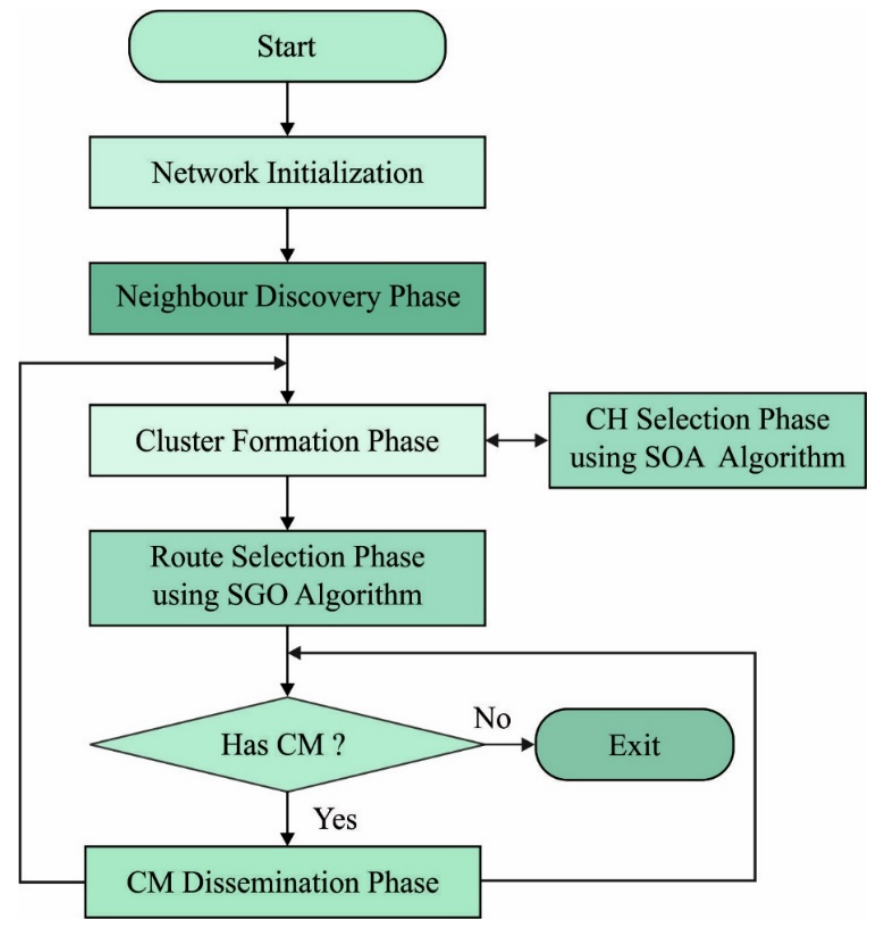

Fig. 2. Workflow of proposed model 


\subsection{Design of SOA-C Technique}

Shark has effective olfactory receptor and capable of finding their prey based on this receptor. This method performs according to the subsequent assumption:

- Injured fish is taken into account as prey for the shark since the fish body distributes blood all over the sea. Also, the injured fish has insignificant speed than the shark.

- The blood is often spread oversea, and the effects of water flow aren't taken into account for the blood distribution.

- Every injured fish is taken into account as single blood production resource for the shark; thus, the olfactory receptor helps sharks to discover their prey

- The first population for sharks is expressed as $\left[X_{1}^{1}, X_{2}^{1}, \ldots, X_{N P}^{1}\right], N P=\operatorname{population}(\operatorname{size})$. Every shark position/solution candidate might contain the subsequent component as follows:

$$
X_{i}^{1}=\left[x_{i, 1^{\prime}}^{1} x_{i, 2^{\prime}}^{1} \ldots, x_{i, N D}^{1}\right],
$$

Whereas $X_{i}^{1}$ represent the first location vector; $x_{i j}^{1}$ denotes the jth parameter of the shark's location; also ND indicates the amount of decision parameters [18]. The first velocity for sharks is expressed as $V_{i}^{1}=$ $\left[v_{i, 1}^{1}, v_{i, 2}^{1}, \ldots, v_{i, N D}^{1}\right]$. the velocity component is taken into account as follows:

$$
V_{i}^{1}=\left[v_{i, 1}^{1}, v_{i, 2}^{1}, \ldots, v_{i, N D}^{1}\right], i=1, \ldots N P,
$$

In which $V_{i}^{1}$ represent the first velocity vector and $v_{i j}^{1}$ indicate the jth parameter of the sharks' velocity. Once the sharks receive higher odor intensity they move fast to their prey. As a result, when the odor intensity is taken into account as an objective function, the velocity change with the variations in the objective function as follows:

$$
V_{i}^{k}=\left.\eta_{k} \cdot R_{1} \cdot \nabla(O F)\right|_{x_{i}^{k}}
$$

Let $\eta_{k}$ be the values among zero \& one; $R_{1}$ represent the arbitrary value, and $O F$ indicate the objective function.

There is inertia in the shark motion that must be taken into account in the shark velocity; therefore, Eq. (3) is altered according to Eq. (4):

$$
v_{i, j}^{k}=\eta_{k} \cdot R_{1} \cdot \frac{\partial(O F)}{\partial x_{j}}+\alpha \cdot R_{2} \cdot v_{i, j}^{k-1},
$$

While $\alpha$ represent the inertia coefficients and $R_{2}$ signifies the arbitrary number among zero \& one. Shark has a particular field for velocity. Their maximal velocity is $80 \mathrm{~km} / \mathrm{hr}$, and their minimal velocity is $20 \mathrm{~km} / \mathrm{hr}$. Therefore, velocity limits are taken into account, and Eq. (4) is altered according to Eq. (5):

$$
\left|v_{i, j}^{k}\right|=\min \left[\left|\eta_{k} \cdot R_{1} \cdot \frac{\partial(O F)}{\partial x_{j}}\right|_{x_{i, j}^{k}}+\alpha_{k} \cdot R_{2} \cdot v_{i, j}^{k-1}|,| \beta_{k} \cdot v_{i, j}^{k-1} \mid\right],
$$

Whereas $\beta_{k}$ represent the velocity limit. Subsequently, the sharks' location is upgraded according to Eq. (6):

$$
Y_{i}^{k+1}=X_{i}^{k}+V_{i}^{k} \Delta t_{k^{\prime}}
$$

In which $\Delta t_{k}$ represent the time step and $Y_{i}^{k+1}$ denotes the novel location for the shark. Shark has rotation motion operators. These operators indicate that shark could escape from the local optimal, also the shark position depends on the rotation motion is altered according to Eq. (7):

$$
Z_{i}^{k+1, m}=Y_{i}^{k+1}+R_{3} \cdot Y_{i}^{k+1}, m=1, \ldots, M,
$$

Let $Z_{i}^{k+1, m}$ be the novel shark location and $M$ represent the amount of local searches for the shark. Three factors are used for the decision of clustering including relative stability (RS), DC, and RMETX. They represent the capacity of vehicles in VANETs.

Initially, calculations and descriptions of the 3 variables were introduced.

The relative stability is determined by the stability of on-hop neighbours. Consider the continuous changes of topology in VANET, relative stability is highly suitable based on the data transmission. It represents when the nodes move relatively slowly/fast/even stable in the neighborhood.

$$
R S_{j}=\sum_{i=1}^{N} d v_{i} \times \frac{1}{N}
$$


In Eq. (8), $N$ represent the numbers of neighbor, also $d v_{-} i$ indicates the relative speed to the neighbouring nodes $i$. Now the $N$ could not be zero when the nodes have no neighbouring node, is the $C H$.

The next variable is calculated according to the numbers of neighbouring nodes and the distance from them, i.e., called DC as given in Eq. (9). The smaller values of DC are selected as it could be anticipated that the nodes are placed nearer would stay longer within one another broadcast range.

$$
D C(i)=\frac{D(i)}{C(i)}
$$

$D(i)$ represent the distance values among nodes $v_{i}$ and its neighbours in the vicinity estimated in Eq. (10). $C(i)$ denotes the connectivity range i.e., the numbers of neighbouring node $v_{i}$.

$$
D(i)=\sum_{j=1, j \neq i}^{N} \sqrt{\left(x_{i}-x_{j}\right)^{2}+\left(y_{i}-y_{j}\right)^{2}}
$$

$\left(x_{i}, y_{i}\right)$ and $\left(x_{j}, y_{j}\right)$ are the coordinates of node $v_{i}$ and $v_{j}$. If a node has more neighbours, the nodes reside in a major location [19]. When CHs have distant members, it is easier to lack the member. Thus, lesser distance summation nodes are selected. The nodes with large amount of neighbours and small distance from neighbours have high possibility to be selected as $\mathrm{CH}$.

ETX predict the numbers of transmission needed to transmit a packet through a connection. Smaller ETX minimizes the overhead, however, reduces the end to end delay by decreasing the collision. The RMETX is an adapted version of ETX which considers the characteristics of VANET. It is a means of reciprocal link ETX $s$ in the node's one-hop neighborhoods. The traditional ETX focus on path/link. The presented RMETX focuses on nodes transmitting capacity.

$$
R M E T X=\frac{\sum_{i=1}^{N} E T X_{i}}{N}
$$

ETX is evaluated using Eq. (12). $N$ represent the numbers of links among the nodes and one-hop neighbours.

$$
\operatorname{ETX}=\frac{1}{d_{f} \cdot d_{r}}
$$

$d_{f}$ represent the forward delivery ratio. $d_{r}$ indicates the reverse delivery ratio. Clearly, $d_{f} \cdot d_{r}$ represent the probability of packet acknowledged and arrives properly.

Assigning different weights have direct influence on the fitness of the nodes to become $\mathrm{CH}$, further, it influences over the life time of $\mathrm{CHS}$.

$$
\text { Wnode }_{i}=w_{R S} R S_{i}+w_{D C} D C_{i}+w_{R M E T X} R M E T X
$$

Integrating this measure, weight factors are attained using Eq. (13), that demonstrates the fitness of nodes to be the $C H$. The small $W$ node $e_{i}$ is, the more capable it is to become $C H$. The values of $w_{R S}, w_{D C}$, and $w_{R M E T X}$ are $0.718,0.187$, and 0.095 respectively.

Each node is grouped into the cluster according to the driving directions. Once the node receives an EMs, initially, it checks either it is a $\mathrm{CH}$. When it isn't a $\mathrm{CH}$ and receives the message at the beginning, it would estimate either its $\mathrm{CH}$ could get this message or not. When the $\mathrm{CH}$ could not get the message, the node would transmit the messages to its $\mathrm{CH}$. When a $\mathrm{CH}$ receives these EMs, it checks either it receives the messages at the beginning or not. Then, it checks either it is placed in the warning zone. When it is outside of the warning zone, it would remove the EMs. When it is inside the warning zone, it checks if there are similar EM events from other directions. Emergency events are recognized by the 1st, 2nd, and 3rd field of the EMs When there are no similar EM events from other directions, it implies hole problem has occurred. The $\mathrm{CH}$ would retransmit the EMs occasionally till it receives similar EM events from other directions.

\subsection{Design of SGO-RDD Technique}

Optimum path problem is the main challenge in effective data dissemination in VANET. This issue aims to determine the optimum path from the source to all remaining nodes in the network with the most stable link containing minimal probability of occurrence of obstacles. The proposed method follows the SGO-RDD technique for routing and data dissemination. This scenario could be determined by complete graph $G=(V, E)$ having a set of vertices $V=\left\{v_{1}, v_{2}, v_{3}, \ldots, v_{n}\right\}$ and set of links $E=\left\{\left(v_{j}, v_{k}: j \neq k,\left(v_{j}, v_{k}\right) \in V\right\}\right.$. Each link is characterized as link stability value (based on angular direction, velocity, and distance) evaluated using Eq. (5) and probability of occurrence of obstacles estimated. These 2 aspects, viz., road obstacles and link stability are the stimulating factors in path selection since the factor results in disconnection problems in vehicular environments [20]. Likewise, vehicles have to change their directions because of the road obstacle that make a higher influence on the stability of link. For optimum path searching, they should be considered a path, i.e., less barrier and more stable connection simultaneously. Thus, the goal is to maximalize the links lifespan from one to other vehicles and minimalize the probability of occurrence of obstacles.

Dhiman G and Kumar V [21] proposed a novel kind of bio-inspired optimization method, the SOA, by investigating the biological features of the seagull. Seagull lives in a group, with their intelligence to attack and 
find their prey. The key features of seagulls are aggressive and migration behaviors. The arithmetical model of the natural behaviors of the seagull is given in the following.

In the migration process, seagull moves from one place to another and met 3 criteria's:

Avoid collision: in order to prevent collision with another seagull, parameter $A$ is used to evaluate the novel location of the search seagulls.

$$
C_{s}(t)=A \times P_{s}(t)
$$

whereas $C_{s}(t)$ represent a novel location which doesn't conflict with another search seagulls, $P_{S}(t)$ represent the present location of the search seagulls, $t$ represents the present iterations and $A$ represents the motional behavior of the search seagulls in the search space.

$$
A=f_{c}-\left(t \times\left(f_{c} / \text { Max }_{\text {iteration }}\right)\right),
$$
$[2,0]$.

Whereas $t=0,1,2, \ldots, \operatorname{Max}_{\text {iteration }}, f_{c}$ could control the frequency of the parameter, and the values drop from

Best position: Afterwards evading overlapping with other seagulls, seagull moves in the direction of optimal location.

$$
M_{s}(t)=B \times\left(P_{b s}(t)-P_{s}(t)\right),
$$

In which $M_{s}(t)$ represent the position of the search seagulls [22]. $B$ denotes the arbitrary value accountable to balance the local and global search seagulls.

$$
B=2 \times A^{2} \times r_{d},
$$

Whereas $r_{d}$ represent an arbitrary value between zero and one.

Close to the best search seagull: Afterward the seagulls move to a location where it doesn't collide with another seagull, it moves in the direction of optimal location to attain its novel location.

Let $D_{s}(t)$ be the optimal fit search seagulls.

$$
D_{s}(t)=\left|C_{s}(t)+M_{s}(t)\right|
$$

Seagulls could always be changing their speed and attack angle at the time of migration. They utilize their weight and wings to retain height. While attacking the prey, they move in a spiral shape in the air. The motional behavior in the $\mathrm{x}, y$, and $z$ planes is determined in the following equation:

$$
\begin{gathered}
x=r \times \cos (\theta), \\
y=r \times \sin (\theta), \\
z=r \times \theta, \\
r=u \times e^{\theta v},
\end{gathered}
$$

Let $r$ be the radius of spiral and $\theta$ denotes an arbitrary angle between $[0,2 \pi] . u \& v$ denotes the relation constant of the spiral shape, and $e$ represents the base of natural logarithm. The attack location of seagull is continuously upgraded.

$$
P_{s}(t)=D_{s}(t) \times x \times y \times z+P_{b s}(t),
$$

In which $P_{S}(t)$ save the optimal solution and update the location of another search seagulls.

Link lifetime $\left(L T_{j, k}\right)$ or Link stability $\left(L S_{j, k}\right)$ specifies the number of times 2 vehicles $j \& k$ remain connected. Assume 2 vehicles $j \& k$ within the transmission range $R$ of one another lies on the two dimensional coordinate systems $\left(x_{j}, y_{j}\right) \&\left(x_{k}, y_{k}\right)$, correspondingly. Likewise, assume vehicles $j \& k$ are moving with direction $\left(\left(\theta_{j}, \theta_{k}\right): 0<\theta_{j}, \theta_{k}<2 \pi\right)$ and speed $\left(v_{j}, v_{k}\right)$. Next, the number of times 2 moving vehicles remain connected is evaluated using Euclidean distance in the polar coordinate scheme. Therefore, the Euclidean distance ' $R$ ' among 2 moving vehicles are:

$$
\begin{aligned}
R^{2}=\left[\left(x_{j}+v_{j} \cos \theta_{j} l_{t}\right)-\left(x_{k}+v_{k} \cos \theta_{k} l_{t}\right)\right]^{2} \\
+\left[\left(y_{j}+v_{j} \sin \theta_{j} l_{t}\right)-\left(y_{k}+v_{k} \sin \theta_{k} l_{t}\right)\right]^{2}
\end{aligned}
$$

In order to evaluate the links lifespan $l_{t}$ where the vehicles $j \& k$ remain connected is calculated by putting $\frac{d R}{d l_{t}}=0$,

$$
l_{t}=\frac{-(a b+c d)}{b^{2}+d^{2}}
$$

Link lifetime $\left(L T_{j, k}\right)$ or Link stability $\left(L S_{j, k}\right)$ of each edge or link is evaluated using Eq. (25).

The inspiration behindhand the problem is to discover an optimum route for data dissemination in dense and sparse networks in the vehicle. For this, they decide to select a path that minimalized the probability of occurrence of obstacles. Assume a theoretical random possibility on every route. Assume $p_{1}, p_{2}, \ldots \ldots, p_{n}$ is the probability of occurrence of obstacles within the network. Next, the overall probability of occurrence of obstacles is $p_{1 \mathrm{n}}=$ $p_{1} \times p_{2} \times \ldots \ldots \times p_{n}$. Logarithmic functions convert the product of probabilities to the amount of the logarithm of probabilities by

$$
\log p_{1 n}=\log p_{1}+\log p_{2}+\cdots \ldots+\log p_{n}
$$

Arithmetically, the minimalization of $\log p_{1 \mathrm{n}}$ is equal to maximization of $-\log p_{1 \mathrm{n}}$, for $\log p_{1 \mathrm{n}} \leq 0$. Consequently, Eq. (26) could be changed into:

$$
-\log p_{1 n}=\left(-\log p_{1}\right)+\left(-\log p_{2}\right)+\cdots+\left(-\log p_{n}\right)
$$


As abovementioned, optimum path problems are determined by the problem of defining optimum path to be utilized for data dissemination from the source to all remaining nodes of VANET. The main purpose is to maximize the link stability and probability of non-occurrence of obstacles, at the same time. Objective or Fitness function is needed for measuring the quality of solution. Consequently, the FF takes maximal values once the optimum route is attained. Therefore, the fitness function is determined by:

$$
\text { fit }_{f}=\text { Maximize } \sum_{k=1}^{n} \sum_{j=1}^{n}\left(L S_{j, k}\right)\left(-\log p_{j k}\right)
$$

Subjected to,

$$
\begin{gathered}
0 \leq p_{j k} \leq 1 \forall j=1,2, \ldots, n ; \forall k=1,2, \ldots \ldots, n \\
L S_{j, k}=L S_{k, j} \forall j=1,2, \ldots, n ; \forall k=1,2, \ldots \ldots, n \\
\sum_{k=1}^{n} \sum_{j=1}^{n} p_{j k}=\sum_{j=1}^{n} \sum_{k=1}^{n} p_{k j}
\end{gathered}
$$

Eq. (29) demonstrates that all the links are related to the probability in range of zero and one, whereas Eq. (30) \& (31) indicates a symmetric problem.

\section{Experimental Validation}

This section examines the performance of the proposed model under several aspects. The parameters involved in the simulation process are given in Table 1 and the results are inspected under distinct dimensions.

\begin{tabular}{|l|l|}
\hline Parameters & Value \\
\hline Simulation region & $1500 \mathrm{~m} * 2000 \mathrm{~m}$ \\
\hline Simulation time & $500 \mathrm{~s}$ \\
\hline No. of repetitions for every run & 10 \\
\hline Vehicle count & $100-500$ \\
\hline Lane count & 2 \\
\hline Vehicle velocity & $20-100 \mathrm{kmph}$ \\
\hline Communication range & $200-350 \mathrm{~m}$ \\
\hline Packet size & $300 \mathrm{bytes}$ \\
\hline Packet interval & $0.01 \mathrm{~s}$ \\
\hline Data Rate & $2 \mathrm{Mbps}$ \\
\hline MAC layer protocol & $802.11 \mathrm{p}$ \\
\hline
\end{tabular}

Table 1. Parameter setting

Table 2 demonstrates the results analysis of the MOC-RCMD technique with existing methods under different vehicle counts.

Fig. 3 portrays the PDR analysis of the MOC-RCMD technique over the other techniques under varying node counts. The figure stated that the MOC-RCMD technique has accomplished improved outcomes with the higher PDR. For instance, with 100 vehicles, the MOC-RCMD technique has attained an increased PDR of $89.07 \%$ whereas the DPSO-VANET, QADD, and GPSR techniques have obtained a reduced PDR of $76.19 \%, 60.03 \%$, and $42.23 \%$. Also, with 300 vehicles, the MOC-RCMD technique has resulted in a higher PDR of $87.70 \%$ whereas the DPSO-VANET, QADD, and GPSR techniques have accomplished a lower PDR of $80.85 \%, 55.65 \%$, and

\begin{tabular}{|c|c|c|c|c|}
\hline No. of Vehicles & MOC-RCMD & DPSO-VANET & QADD & GPSR \\
\hline \multicolumn{5}{|l|}{ PDR (\%) } \\
\hline 100 & 89.07 & 76.19 & 60.03 & 42.23 \\
\hline 200 & 89.89 & 83.59 & 73.45 & 64.42 \\
\hline 300 & 87.70 & 80.85 & 55.65 & 55.38 \\
\hline 400 & 83.59 & 71.26 & 43.32 & 58.39 \\
\hline 500 & 75.10 & 63.87 & 40.04 & 58.94 \\
\hline
\end{tabular}
$55.38 \%$. Besides, with 500 vehicles, the MOC-RCMD technique has demonstrated a maximum PDR of $75.10 \%$ whereas the DPSO-VANET, QADD, and GPSR techniques have exhibited a minimal PDR of 63.87\%, 40.04\%, and $58.94 \%$. 


\begin{tabular}{|c|c|c|c|c|}
\hline \multicolumn{5}{|c|}{ Average Throughput (kbps) } \\
\hline 100 & 216.31 & 200.56 & 143.28 & 87.44 \\
\hline 200 & 191.97 & 164.05 & 123.96 & 106.06 \\
\hline 300 & 161.90 & 112.50 & 103.19 & 107.49 \\
\hline 400 & 165.48 & 137.56 & 99.61 & 93.89 \\
\hline 500 & 167.63 & 121.09 & 99.61 & 95.32 \\
\hline \multicolumn{5}{|c|}{ Routing Overhead (\%) } \\
\hline 100 & 16.82 & 23.62 & 35.31 & 37.76 \\
\hline 200 & 21.71 & 34.77 & 33.41 & 37.22 \\
\hline 300 & 20.35 & 31.78 & 37.49 & 49.19 \\
\hline 400 & 21.99 & 36.40 & 48.10 & 50.00 \\
\hline 500 & 25.52 & 39.67 & 53.27 & 53.81 \\
\hline \multicolumn{5}{|c|}{ End to End Delay (ms) } \\
\hline 100 & 98.92 & 105.73 & 109.14 & 127.88 \\
\hline 200 & 143.21 & 161.95 & 182.39 & 190.91 \\
\hline 300 & 209.65 & 228.39 & 245.43 & 270.98 \\
\hline 400 & 281.20 & 303.35 & 347.64 & 374.90 \\
\hline 500 & 405.56 & 439.63 & 456.67 & 519.70 \\
\hline
\end{tabular}

Table 2. Results analysis of MOC-RCMD technique under varying vehicle count

Fig. 4 depicts the ATT analysis of the MOC-RCMD technique with existing approaches under variable node count. The figure specified that the MOC-RCMD technique has gained enhanced performance with the improved ATT. For instance, with 100 vehicles, the MOC-RCMD technique has provided a raised ATT of $216.31 \mathrm{kbps}$ whereas the DPSO-VANET, QADD, and GPSR techniques have accomplished a decreased ATT of $200.56 \mathrm{kbps}$, $143.28 \mathrm{kbps}$, and $87.44 \mathrm{kbps}$. Meanwhile, with 300 vehicles, the MOC-RCMD technique has led to an increased ATT of $161.90 \mathrm{kbps}$ whereas the DPSO-VANET, QADD, and GPSR techniques have resulted in a reduced ATT of $112.50 \mathrm{kbps}, 103.19 \mathrm{kbps}$, and $107.49 \mathrm{kbps}$. Eventually, with 500 vehicles, the MOC-RCMD technique has established a supreme ATT of $167.63 \mathrm{kbps}$ whereas the DPSO-VANET, QADD, and GPSR techniques have displayed a negligible ATT of $121.09 \mathrm{kbps}, 99.61 \mathrm{kbps}$, and $95.32 \mathrm{kbps}$.

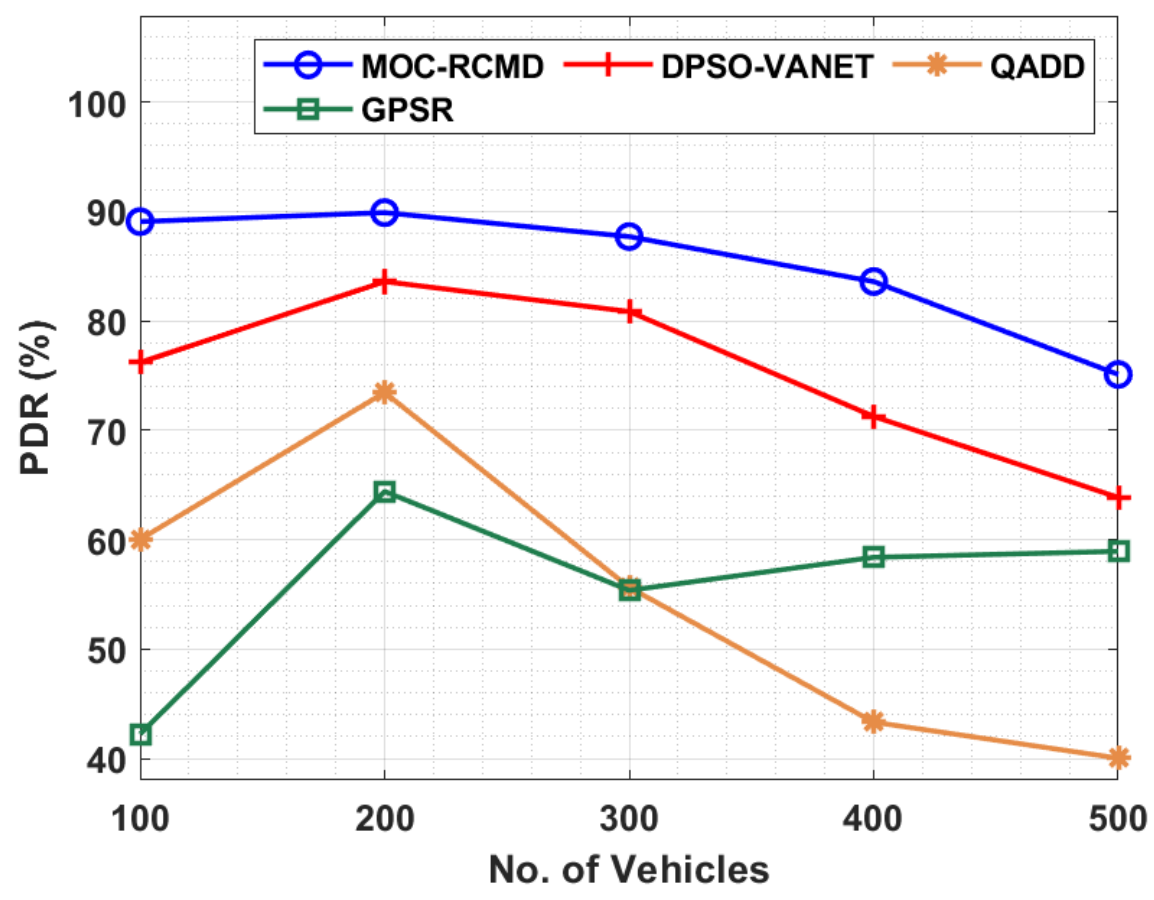

Fig. 3. PDR analysis of MOC-RCMD model with different vehicle count

A comparative routing overhead (RO) analysis of the MOC-RCMD technique is made in Fig. 5 and the results reported the betterment of the MOC-RCMD technique with the least RO under all vehicle count. For instance, under 100 vehicles, the MOC-RCMD technique has achieved better results with the RO of $16.82 \%$ whereas the 
DPSO-VANET, QADD, and GPSR techniques have occasioned a poor outcome with the higher RO of $23.62 \%$, $35.31 \%$, and $37.76 \%$ respectively. Followed by, under 500 vehicles, the MOC-RCMD technique has realized better results with the RO of $25.52 \%$ whereas the DPSO-VANET, QADD, and GPSR techniques have failed to gain considerable performance with the increased $\mathrm{RO}$ of $39.67 \%, 53.27 \%$, and $53.81 \%$ respectively.

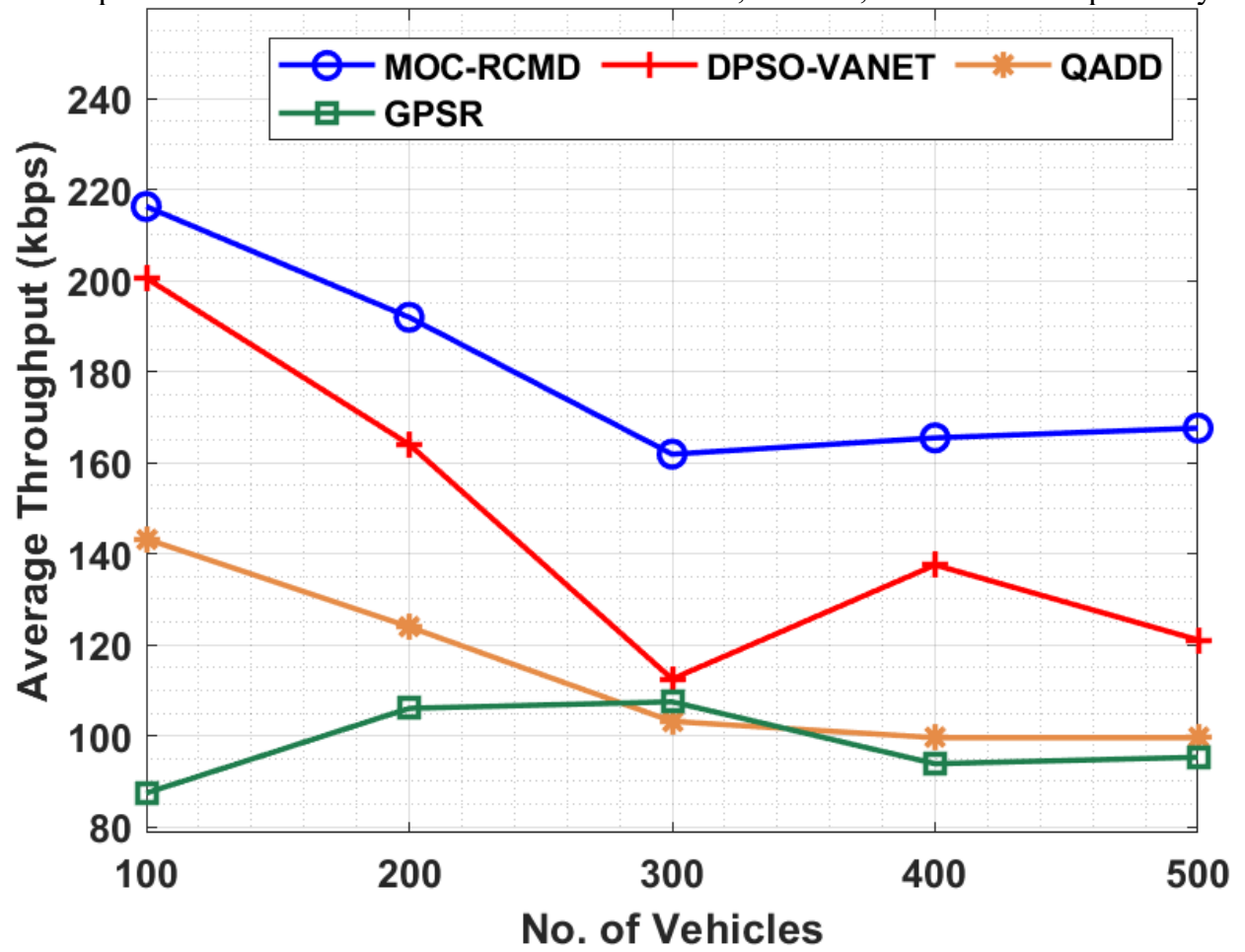

Fig. 4. ATT analysis of MOC-RCMD model with different vehicle count

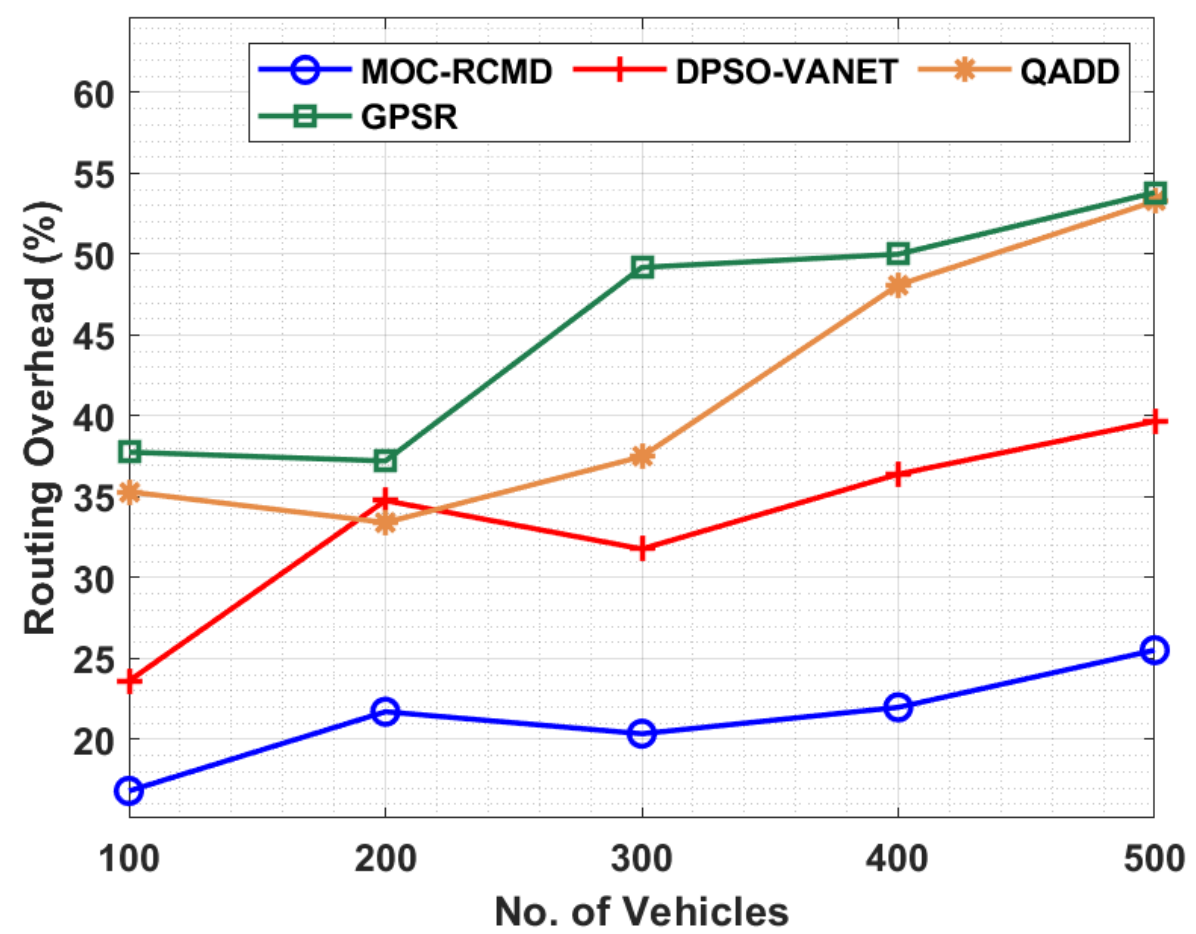

Fig. 5. RO analysis of MOC-RCMD model with different vehicle count

A detailed comparative study of the MOC-RCMD technique interms of ETED is performed in Fig. 6. The figure revealed that the MOC-RCMD technique has outperformed the existing ones with the minimal ETED. For instance, under 100 vehicles, the MOC-RCMD technique has realized better results with the ETED of 98.92ms whereas the DPSO-VANET, QADD, and GPSR techniques have reached ineffective results with the ETED of $105.73 \mathrm{~ms}, 109.14 \mathrm{~ms}$, and $127.88 \mathrm{~ms}$ respectively. Furthermore, under 500 vehicles, the MOC-RCMD technique has realized better results with the ETED of $405.56 \mathrm{~ms}$ whereas the DPSO-VANET, QADD, and GPSR techniques 
become unsuccessful to attain significant outcomes with the maximum ETED of $439.63 \mathrm{~ms}, 456.67 \mathrm{~ms}$, and $519.70 \mathrm{~ms}$ respectively.

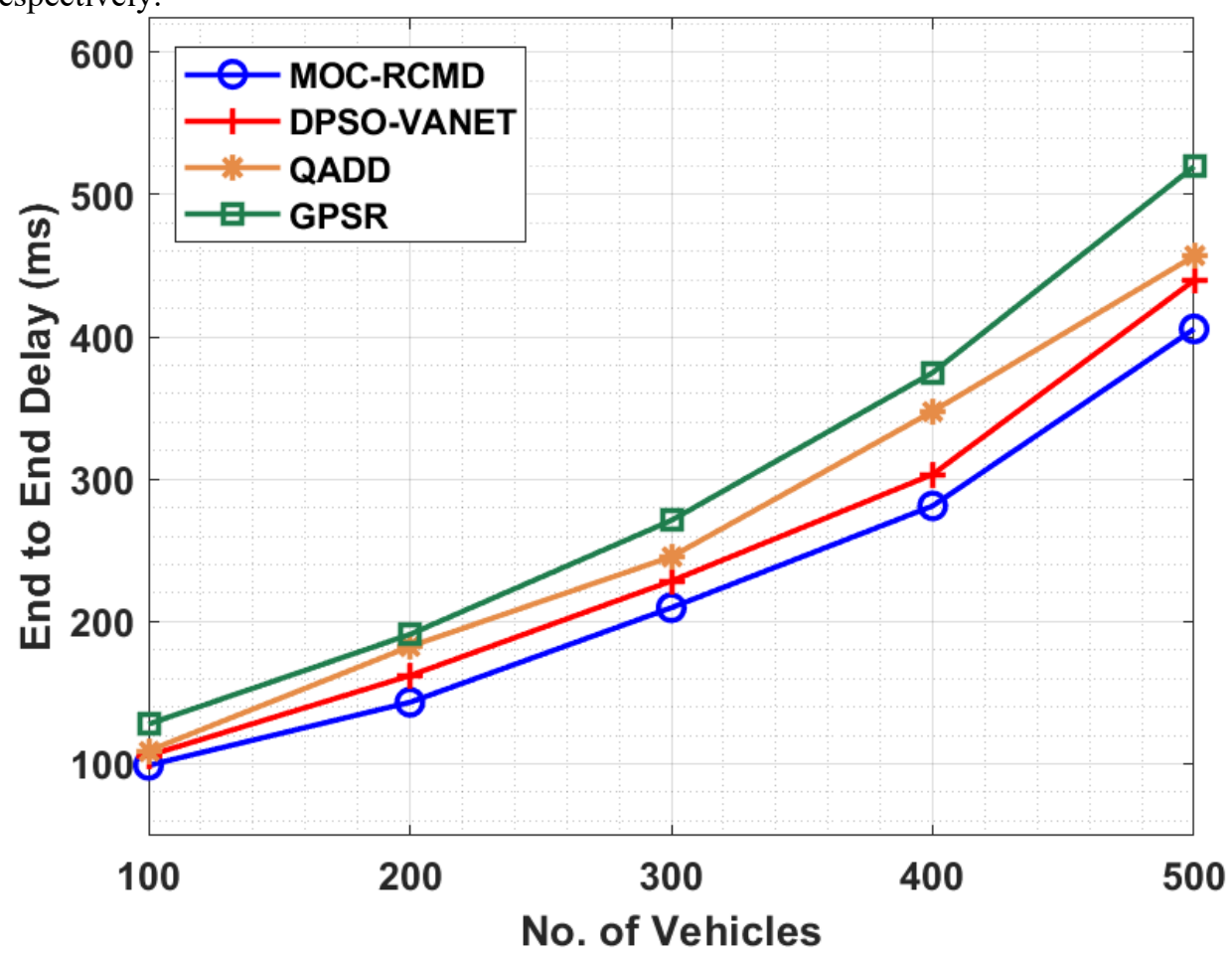

Fig. 6. ETED analysis of MOC-RCMD model with different vehicle count

\section{Conclusion}

In this study, a new MOC-RCMD technique is derived to disseminate CLM in VANET. The proposed MOCRCMD technique involves two-stage process namely SOA-C based cluster construction and SGO-RDD based Data Dissemination. The SOA-C and SGO-RDD techniques derive a fitness function involving different input parameters related to VANET. The proposed MOC-RCMD technique has the capability to organize the clusters and select CHs proficiently. In order to showcase the better performance of the proposed model, a series of experimental analyses are carried out and the results are investigated interms of several measures. The simulation results highlighted the betterment of the MOC-RCMD technique over the recent approaches. In future, data aggregation approaches can be included to reduce the quantity of data communication in VANET.

\section{References}

[1] A.-M. Ahmad, H. Idriss, A. E. Mouallem, and Z. E. Bazzal, "Chain-based data dissemination in vehicular ad-hoc networks (VANETs)," in Proc. 6th Int. Conf. Digit. Inf., Netw., Wireless Commun. (DINWC), Apr. 2018, pp. 75-80.

[2] S. R. Kolte and M. S. Madnkar, "A design approach of congestion control for safety critical message transmission in VANET," in Proc. 4th Int. Conf. Commun. Syst. Netw. Technol., Apr. 2014, pp. 298-301.

[3] T. Qiu, R. Qiao, and D. O. Wu, "EABS: An event-aware backpressure scheduling scheme for emergency internet of things," IEEE Trans. Mobile Comput., vol. 17, no. 1, pp. 72-84, Jan. 2018.

[4] Ghazi, M.U.; Khattak, M.A.K.; Shabir, B.; Malik, A.W.; Ramzan, M.S. Emergency message dissemination in vehicular networks: A review. IEEE Access 2020, 8, 38606-38621.

[5] Haider, S.; Abbas, G.; Abbas, Z.H.; Baker, T. DABFS: A robust routing protocol for warning messages dissemination in VANETs. Comput. Commun. 2019, 147, 21-34.

[6] Sun, Y.; Kuai, R.; Xiao, S.; Tang, W.; Li., X. VIMAC: Vehicular information medium access control protocol for high reliable and low latency transmissions for vehicular ad hoc networks in smart city. Future Gener. Comput. Syst. 2020, 106, 55-66.

[7] Mchergui, A.; Moulahi, T.; Othman, M.T.B.; Nasri, S. Enhancing VANETs broadcasting performance with mobility prediction for smart road. Wirel. Pers. Commun. 2020, 112, 1629-1641.

[8] Liu, L.; Chen, C.; Qiu, T.; Zhang, M.; Li, S.; Zhou, B. A data dissemination scheme based on clustering and probabilistic broadcasting in VANETs. Veh. Commun. 2018, 13, 78-88.

[9] Haider, S.; Abbas, G.; Abbas, Z.H.; Boudjit, S.; Halim, Z. P-DACCA: A probabilistic direction-aware cooperative collision avoidance scheme for VANETs. Future Gener. Comput. Syst. 2020, 103, 1-17.

[10] Ullah, A., Yaqoob, S., Imran, M. and Ning, H., 2018. Emergency message dissemination schemes based on congestion avoidance in VANET and vehicular FoG computing. IEEE Access, 7, pp.1570-1585.

[11] Benkerdagh, S. and Duvallet, C., 2019. Cluster-based emergency message dissemination strategy for VANET using V2V communication. International Journal of Communication Systems, 32(5), p.e3897.

[12] Shah, S.S., Malik, A.W., Rahman, A.U., Iqbal, S. and Khan, S.U., 2019. Time barrier-based emergency message dissemination in vehicular ad-hoc networks. IEEE Access, 7, pp.16494-16503. 
[13] Ali, M., Malik, A.W., Rahman, A.U., Iqbal, S. and Hamayun, M.M., 2019. Position-based emergency message dissemination for Internet of vehicles. International Journal of Distributed Sensor Networks, 15(7), p.1550147719861585.

[14] Liu, Z., Weng, J., Ma, J., Guo, J., Feng, B., Jiang, Z. and Wei, K., 2019. TCEMD: A trust cascading-based emergency message dissemination model in VANETs. IEEE Internet of Things Journal, 7(5), pp.4028-4048.

[15] Ullah, S., Abbas, G., Abbas, Z.H., Waqas, M. and Ahmed, M., 2020. RBO-EM: Reduced broadcast overhead scheme for emergency message dissemination in VANETs. IEEE Access, 8, pp.175205-175219.

[16] Ullah, S., Abbas, G., Waqas, M., Abbas, Z.H., Tu, S. and Hameed, I.A., 2021. EEMDS: An Effective Emergency Message Dissemination Scheme for Urban VANETs. Sensors, 21(5), p.1588.

[17] Arora, P.O., 2018, April. Reliable emergency message dissemination in VANET using hybrid partitioning approach. In 2018 International Conference on Communication and Signal Processing (ICCSP) (pp. 0672-0677). IEEE.

[18] Yaseen, Z.M., Ehteram, M., Hossain, M., Fai, C.M., Binti Koting, S., Mohd, N.S., Binti Jaafar, W.Z., Afan, H.A., Hin, L.S., Zaini, N. and Ahmed, A.N., 2019. A novel hybrid evolutionary data-intelligence algorithm for irrigation and power production management: application to multi-purpose reservoir systems. Sustainability, 11(7), p.1953.

[19] Jin, D., Yang, J. and Song, J., 2017. Parallel Swarm Optimization Based Cluster for Emergency Message Dissemination in VANETs. Journal of Telecommunication, Electronic and Computer Engineering (JTEC), 9(2-4), pp.77-82.

[20] Chahal, M. and Harit, S., 2019. Optimal path for data dissemination in Vehicular Ad Hoc Networks using meta-heuristic. Computers \& Electrical Engineering, 76, pp.40-55.

[21] Dhiman, G.; Kumar, V. Seagull optimization algorithm: Theory and its applications for large-scale industrial engineering problems. Knowl.-Based Syst. 2019, 165, 169-196.

[22] Chen, X., Li, Y., Zhang, Y., Ye, X., Xiong, X. and Zhang, F., 2021. A Novel Hybrid Model Based on An Improved Seagull Optimization Algorithm for Short-Term Wind Speed Forecasting. Processes, 9(2), p.387.

\section{Authors Profile}

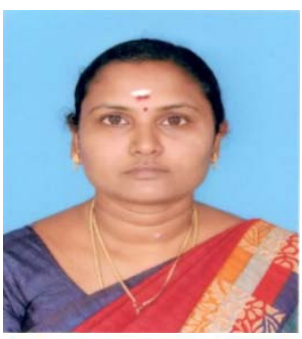

S. Parameswari received her M.Tech degree in Information Technology from Sathayabama University, India in 2006. Now she is a PhD student in the department of Computer Science and Engineering in Annamalai University. Her research interest includes Vehicular Adhoc Networks, Wireless Networks and Web services.

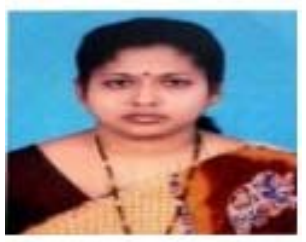

Dr.K.Kavitha received her B.E degree in Computer Science and Engineering and M.E degree in Computer Science and Engineering in 1998 and 2007 respectively. She received her PhD degree in Computer Science from Annamalai University in 2014. She is currently an Associate Professor in Computer Science and Engineering department, Annamalai University. Her research interest includes Adhoc network, Vehicular networks, Sensor networks and IoT.

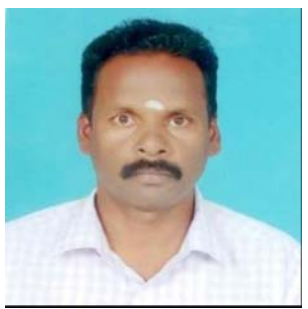

Dr. P.Elango has completed Master of Technology from National Institute of Technology Karnataka (NITK), India and PhD from National Institute of Technology, Tiruchirappalli, Tamil Nadu, India. Presently he is working in Perunthalaivar Kamarajar Institute of Engineering and Technology (PKIET) (Govt. of Puducherry Institution) since 2007. Also, he has published many research papers in various reputed journals. His Research interests are Image processing, Data Mining and Adhoc Network. 